\title{
Microwave Fourier Transform Double Resonance Experiment and Theory
}

\author{
H. Dreizler, E. Fliege, H. Mäder, and W. Stahl \\ Abteilung Chemische Physik im Institut für Physikalische Chemie der Universität Kiel
}

Z. Naturforsch. 37a, 1266-1271 (1982); received August 28, 1982

We present the first experience with a three-level double resonance experiment performed with a microwave Fourier transform spectrometer. The results are described by a theoretical treatment based on three-level Bloch equations.

\section{Introduction}

Battaglia, Gozzini, and Polacco [1], Shimoda, Yajima [2, 3], and Cox, Flynn, and Wilson [4] supplemented microwave spectroscopy by double resonance and thereby increased the versatility and applicability of this field of spectroscopy. In the course of our efforts to develop microwave Fourier transform spectroscopy (MWFT) of molecular gases in thermal equilibrium [5-8], we tried to test whether double resonance experiments are possible with this technique.

\section{Experimental}

To perform a double resonance experiment, it is necessary that two radiations interact with the molecular ensemble. A signal radiation detects the changes introduced by a usually strong pump radiation, which is resonant or near resonant to a transition as shown in Figure 1. It is essential that the pump radiation is prevented from reaching the detection system, i.e. the performance of the MWFT spectrometer as described earlier [6] should not be disturbed.

These conditions could be fulfilled in the first place by replacing the waveguide to coax transition

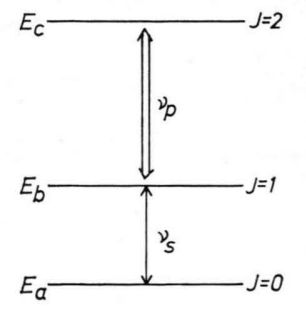

Fig. 1. System of three level double resonance, here Carbonylsulfide, OCS, $\Leftrightarrow$ pump radiation, $\leftrightarrow$ signal radiation, $v_{\mathrm{s} 0}=\left(E_{\mathrm{b}}-E_{\mathrm{a}}\right) / h ; \quad v_{\mathrm{p} 0}=$ $\left(E_{\mathrm{c}}-E_{\mathrm{b}}\right) / h$.

Reprint requests to Prof. Dr. H. Dreizler, Institut für Physikalische Chemie, Olshausenstr. 40-60, D-2300 Kiel. 9a of Fig. 1 in Ref. [6] by a special tunable transition, on the back side of which an R-band waveguide (26.4-40 GHz, $22 \mathrm{GHz}$ cut off) is introduced. Through this the pump radiation is fed into the sample cell of $23 \times 23 \mathrm{~mm}$ cross section (see Figure 2 ). The pump radiation is supplied by a phase stabilized BWO and is amplified by a TWTA. A maximum of $2 \mathrm{~W}$ was available. Secondly we put a band and low pass filter in front of the switch 23 of Fig. 1 in Ref. [6]. Carbonylsulfide, ${ }^{16} \mathrm{O}^{12} \mathrm{C}^{32} \mathrm{~S},{ }^{16} \mathrm{O}^{12} \mathrm{C}^{34} \mathrm{~S}$, and ${ }^{18} \mathrm{O}^{12} \mathrm{C}^{32} \mathrm{~S}$ in natural abundancies were used for this test. As can be seen from Fig. $3-7$ a double resonance doublet was observed. The behaviour of the doublet with variation of pump frequency, pump power and gas pressure reminds of the results from

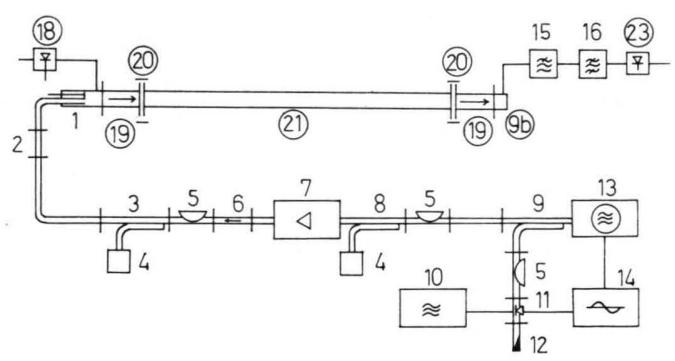

Fig. 2. Changes of the MWFT spectrometer X-band for double resonance experiments. Compare Fig. 1 [6]. Numbers in circles refer to Fig. 1 [6]. 1 Tunable waveguide to coax transition $\mathrm{X}$-band with inserted $\mathrm{R}$-band waveguide (26.4-40 GHz); 2 Transition K- to R-band; 3 Directional coupler $30 \mathrm{~dB}$; 4 Power meter; 5 Attenuator; 6 Waveguide isolator; 7 TWT-amplifier K-band, Hughes $1077 \mathrm{H}, 1 \mathrm{~W}$ nominal; 8 Directional coupler $20 \mathrm{~dB}$; 9 Directional coupler $10 \mathrm{~dB}$; 10 Frequency standard, Rhode und Schwarz, XUC; 11 Mixer; 12 Termination; 13 Backward wave oscillator, Hewlett Packard 8690 B with 8696 A; 14 Synchronizer, Schomandl FDS $30 ; 15$ Low pass filter, cut off $14 \mathrm{GHz}$, K \& L FLR-14, rejection $20 \mathrm{~dB}$ at $15.4 \mathrm{GHz}$, insertion loss $0.5 \mathrm{~dB}$, VSWR $2: 1 ; 16$ Band pass filter, $8-12 \mathrm{GHz}$, Filtronics F 10021 , rejection $>60 \mathrm{~dB}$ for 14 to $18 \mathrm{GHz}$, insertion loss $0.75 \mathrm{~dB}$, VSWR 1.5:1. 

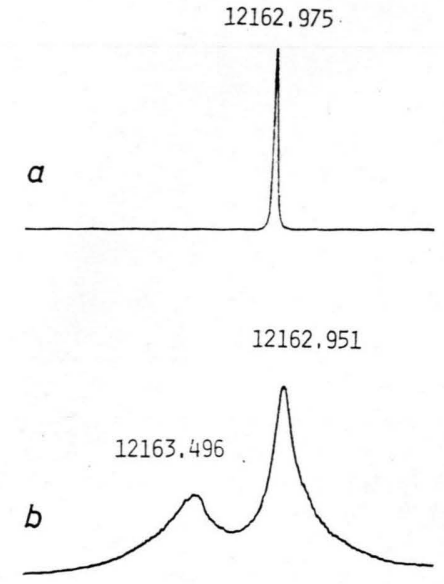

12163.020

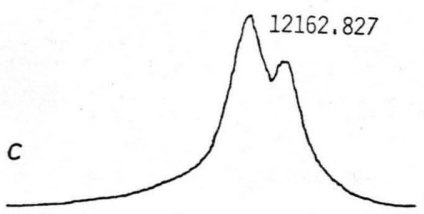

Fig. 3. A range of $2.5 \mathrm{MHz}$ out of a $5 \mathrm{MHz}$ scan of the rotational spectrum of OCS, line $J=1-0$ sample interval $50 \mathrm{~ns}, 1024$ data points extended to 4096 by zeros, spectral point distance $5 \mathrm{kHz}$, signals normalized, frequencies in $\mathrm{MHz}$. a) 500000 averaging cycles, without pump radiation, 0.3 mtorr, $-3{ }^{\circ} \mathrm{C}$; b) 1250000 averaging cycles, pump frequency at $24325.501 \mathrm{MHz}, 1.8 \mathrm{~W}$ at cell entrance, 0.3 mtorr, $-3{ }^{\circ} \mathrm{C}$; c) 750000 averaging cycles, pump frequency at $24326.001 \mathrm{MHz}, 1.8 \mathrm{~W}, 0.3 \mathrm{mtorr},-30^{\circ} \mathrm{C}$.

steady-state double resonance experiments. However, the experimental conditions are somewhat different. In MWFT spectroscopy a transient emission following a strong polarizing $\pi / 2$-pulse is observed. For further details see the following section.

It may be pointed out that the high resolution of MWFT spectroscopy is very useful for resolving the doublet. Using the symmetry of the doublet we were able to adjust the pump frequency to $\pm 1 \mathrm{kHz}$ for the transition $J=2-1$ of ${ }^{16} \mathrm{O}^{12} \mathrm{C}^{32} \mathrm{~S}$ to $24325.927 \mathrm{MHz}$. A frequency of $24325.930(20) \mathrm{MHz}$ was reported [9].

\section{Theory}

A sufficient model to describe the experimental findings given above is that of a three level system with the energy levels $E_{\mathrm{a}}<E_{\mathrm{b}}<E_{\mathrm{c}}$ and the two dipole-allowed transitions between $E_{\mathrm{a}}$ and $E_{\mathrm{b}}$ (sig-

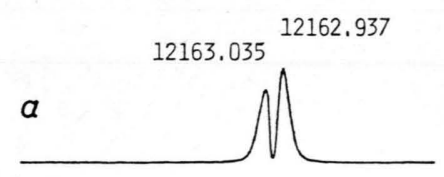

$12163.028 \quad 12162.930$

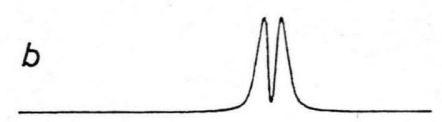

$12163.024 \quad 12162.929$

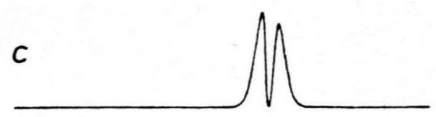

d

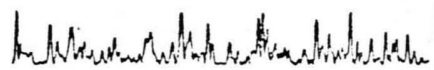

Fig. 4. Adjustment of the pump frequency (general conditions see Fig. 3) 500000 averaging cycles, 0.3 mtorr, $-59^{\circ} \mathrm{C}, 50 \mathrm{~mW}$ at cell entrance. a) pump frequency at $24325.920 \mathrm{MHz}$; b) pump frequency at $24325.927 \mathrm{MHz}$; c) pump frequency at $24325.930 \mathrm{MHz}$; d) 250000 averaging cycles, pump frequency at $24325.901 \mathrm{MHz}, 1 \mathrm{~W}$ at cell entrance, no substance.

nal transition) and between $E_{\mathrm{b}}$ and $E_{\mathrm{c}}$ (pump transition). The following theoretical treatment is based on the three-level Bloch equations formalism, which has been derived earlier by Feuillade et al. [10]. In contrast to the normal description of steady-state double resonance experiments [11] (assuming high pump and low probe microwave power), transient solutions of the Bloch equations will be considered here to describe the behaviour of the system after a high power signal pulse excitation in the presence of a high power pump radiation.

For our experimental setup the microwave radiation is given by a superposition of pump and signal microwave radiation, plane polarized along the same direction (parallel fields)

$$
\varepsilon=2 \varepsilon_{\mathrm{p}} \cos \omega_{\mathrm{p}} t+2 \varepsilon_{\mathrm{s}} \cos \omega_{\mathrm{s}} t,
$$

where $\varepsilon_{\mathrm{p}}$ and $\varepsilon_{\mathrm{s}}$ denote the electric field amplitude of the pump and signal radiation with angular frequencies $\omega_{\mathrm{p}}$ and $\omega_{\mathrm{s}}$ respectively. With (1) and inclusion of intermolecular interaction (relaxation) 


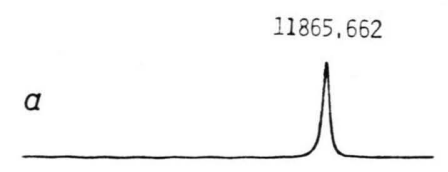

$11065.778 \quad 11865,547$

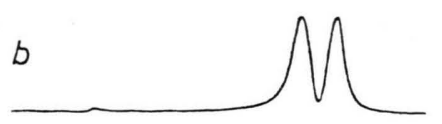

$11865.744 \quad 11865.580$

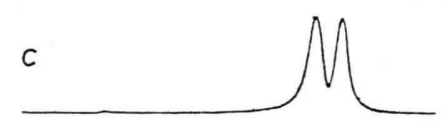

11865.73011865 .591

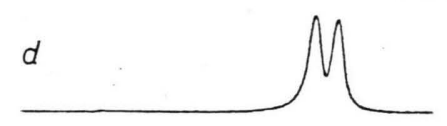

11865.71311865 .605

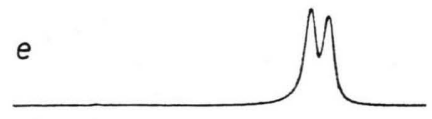

11865.70511865 .611

$f$

Fig. 5. Pump power variation (conditions see Fig. 3) line $J=1-0$ of ${ }^{16} \mathrm{O}^{12} \mathrm{C}^{34} \mathrm{~S}, 250000$ averaging cycles, pump frequency at $23731.302 \mathrm{MHz}, 3$ mtorr, $-59^{\circ} \mathrm{C}$; pump power at cell entrance: a) $0 \mathrm{~mW}$; b) $20 \mathrm{~mW}$; c) $10 \mathrm{~mW}$; d) $5 \mathrm{~mW}$; e) $2 \mathrm{~mW}$; f) $1 \mathrm{~mW}$.

under the impact approximation, "three-level Bloch equations" have been derived by considering the equation of motion of the density matrix for the three-level system [10]. The time dependent behaviour of the variables describing the ensemble of three-level systems is then given by a set of coupled differential equations

$$
\begin{aligned}
\dot{U}_{\mathrm{s}}= & -\Delta \omega_{\mathrm{s}} V_{\mathrm{s}}+\left(x_{\mathrm{p}} / 2\right) V-\left(1 / T_{2}^{\mathrm{ab}}\right) U_{\mathrm{s}} \\
\dot{V}_{\mathrm{s}}= & \Delta \omega_{\mathrm{s}} U_{\mathrm{s}}-x_{\mathrm{s}} W_{\mathrm{s}} \\
& -\left(x_{\mathrm{p}} / 2\right) U-\left(1 / T_{2}^{\mathrm{ab}}\right) V_{\mathrm{s}}
\end{aligned}
$$

11865.70511865 .611

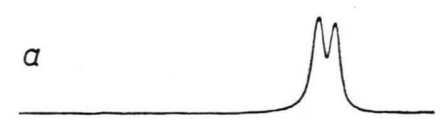

11865.71111865 .604

$b$

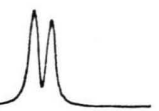

Fig. 6. Gas pressure variation (conditions see Fig. 3) line $J=1-0$ of ${ }^{16} \mathrm{O}^{12} \mathrm{C}^{34} \mathrm{~S}, 250000$ averaging cycles, pump frequency at $23731.302 \mathrm{MHz}$, pump power $1 \mathrm{~mW}$ at cell entrance, $-59^{\circ} \mathrm{C}$ : a) $3 \mathrm{mtolr}$; b) $0.85 \mathrm{mtorr}$.

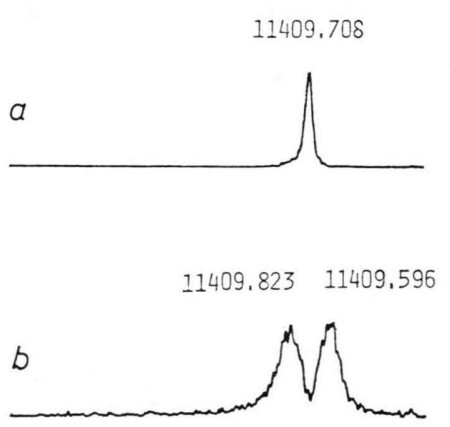

Fig. 7. Double resonance signal of ${ }^{18} \mathrm{O}^{12} \mathrm{C}^{32} \mathrm{~S}$, line $J=1-0$ (conditions see Figure 3). 750000 averaging cycles: a) without pump; b) pump frequency at $22819.404 \mathrm{MHz}$, pump power $20 \mathrm{~mW}$ at cell entrance; $3 \mathrm{mtorr} ;-59^{\circ} \mathrm{C}$.

$$
\begin{aligned}
\dot{W}_{\mathrm{s}}= & x_{\mathrm{s}} V_{\mathrm{s}}-\left(x_{\mathrm{p}} / 2\right) V_{\mathrm{p}} \\
& -\gamma_{y}\left(W_{\mathrm{s}}-W_{\mathrm{s}}^{0}\right)-\gamma_{z}\left(W_{\mathrm{p}}-W_{\mathrm{p}}^{0}\right) \\
\dot{U}_{\mathrm{p}}= & -\Delta \omega_{\mathrm{p}} V_{\mathrm{p}}-\left(x_{\mathrm{s}} / 2\right) V-\left(1 / T_{2}^{\mathrm{bc}}\right) U_{\mathrm{p}} \\
\dot{V}_{\mathrm{p}}= & \Delta \omega_{\mathrm{p}} U_{\mathrm{p}}-x_{\mathrm{p}} W_{\mathrm{p}}-\left(x_{\mathrm{s}} / 2\right) U \\
& -\left(1 / T_{2}^{\mathrm{bc}}\right) V_{\mathrm{p}} \\
\dot{W}_{\mathrm{p}}= & x_{\mathrm{p}} V_{\mathrm{p}}-\left(x_{\mathrm{s}} / 2\right) V_{\mathrm{s}}-\gamma_{x}\left(W_{\mathrm{p}}-W_{\mathrm{p}}^{0}\right) \\
& -\gamma_{\mathrm{w}}\left(W_{\mathrm{s}}-W_{\mathrm{s}}^{0}\right) \\
\dot{U}= & -\Delta \omega V-\left(x_{\mathrm{s}} / 2\right) V_{\mathrm{p}}+\left(x_{\mathrm{p}} / 2\right) V_{\mathrm{s}} \\
& -\left(1 / T_{2}^{\mathrm{ac}}\right) U \\
\dot{V}= & \Delta \omega U+\left(x_{\mathrm{s}} / 2\right) U_{\mathrm{p}}-\left(x_{\mathrm{p}} / 2\right) U_{\mathrm{s}} \\
& -\left(1 / T_{2}^{\mathrm{ac}}\right) V .
\end{aligned}
$$

The variables in (2) are related to the elements of the density matrix $\tilde{\varrho}_{i j}(i, j \in\{\mathrm{a}, \mathrm{b}, \mathrm{c}\})$ in the interaction representation as follows.

$$
\begin{aligned}
& W_{\mathrm{s}}=\tilde{\varrho}_{\mathrm{aa}}-\tilde{\varrho}_{\mathrm{bb}}, \\
& W_{\mathrm{p}}=\tilde{\varrho}_{\mathrm{bb}}-\tilde{\varrho}_{\mathrm{cc}},
\end{aligned}
$$

the differences of occupation probabilities between the energy levels of signal and pump transition with 
thermal equilibrium values $W_{\mathrm{s}}{ }^{0}$ and $W_{\mathrm{p}}{ }^{0}$ respectively, and

$$
\begin{aligned}
U_{\mathrm{s}} & =\tilde{\varrho}_{\mathrm{ab}}+\tilde{\varrho}_{\mathrm{ba}}, \\
V_{\mathrm{s}} & =i\left(\tilde{\varrho}_{\mathrm{ba}}-\tilde{\varrho}_{\mathrm{ab}}\right), \\
U_{\mathrm{p}} & =\tilde{\varrho}_{\mathrm{bc}}+\tilde{\varrho}_{\mathrm{cb}}, \\
V_{\mathrm{p}} & =i\left(\tilde{\varrho}_{\mathrm{cb}}-\tilde{\varrho}_{\mathrm{bc}}\right), \\
U & =\tilde{\varrho}_{\mathrm{ac}}+\tilde{\varrho}_{\mathrm{ca}}, \\
V & =i\left(\tilde{\varrho}_{\mathrm{ca}}-\tilde{\varrho}_{\mathrm{ac}}\right),
\end{aligned}
$$

corresponding to a superposition of states for the energy level pairs $\left(E_{\mathrm{a}}, E_{\mathrm{b}}\right),\left(E_{\mathrm{b}}, E_{\mathrm{c}}\right)$ and $\left(E_{\mathrm{a}}, E_{\mathrm{c}}\right)$ respectively. The quantities $U_{\mathrm{s}}\left(U_{\mathrm{p}}\right)$ and $V_{\mathrm{s}}\left(V_{\mathrm{p}}\right)$ are proportional to the in- and out-of-phase component of the macroscopic polarisation with respect to the external signal (pump) microwave radiation [12], in contrast to $U$ and $V$ which do not correspond to a dipole polarization as the transition dipole matrix element $\mu_{\mathrm{ac}}$ is assumed to be zero. The other symbols used in (2) are

$$
\begin{aligned}
\Delta \omega_{\mathrm{s}} & =\left(E_{\mathrm{b}}-E_{\mathrm{a}}\right) / \hbar-\omega_{\mathrm{s}}, \\
\Delta \omega_{\mathrm{p}} & =\left(E_{\mathrm{c}}-E_{\mathrm{b}}\right) / \hbar-\omega_{\mathrm{p}}, \\
\Delta \omega & =\Delta \omega_{\mathrm{s}}+\Delta \omega_{\mathrm{p}}
\end{aligned}
$$

the deviations of the incident radiations from resonance, and

$$
\begin{aligned}
& x_{\mathrm{s}}=\left(2 \mu_{\mathrm{ab}} / \hbar\right) \varepsilon_{\mathrm{s}}, \\
& x_{\mathrm{p}}=\left(2 \mu_{\mathrm{bc}} / \hbar\right) \varepsilon_{\mathrm{p}},
\end{aligned}
$$

the Rabi frequencies of signal and pump transition with the dipole matrix elements $\mu_{\mathrm{ab}}$ and $\mu_{\mathrm{bc}}$ respectively. To allow for collisional relaxation a set of relaxation parameters for the different elements of the density matrix is introduced into the equations where $\gamma_{x}, \gamma_{y}, \gamma_{z}$ and $\gamma_{\mathrm{w}}$ concern the relaxation of population (see [10] for definition), and $T_{2}^{\mathrm{ab}}, T_{2}^{\mathrm{bc}}$ and $T_{2}^{\text {ac }}$ are the relaxation times for the superposition of the indicated pairs of levels.

We now proceed and discuss the solutions of (2) as applied to the Fourier transform double resonance experiment described in this paper. According to the experimental procedure the pump microwave radiation is assumed to continuously irradiate the sample gas. The time interval between the signal microwave pulses of duration $t_{1}$ is considered to be long with respect to the relaxation times so that it is sufficient to describe the dynamical behaviour of the system by a single pulse excitation. Identi- fying the start of the signal microwave pulse by $t=0$ we then have

$$
\begin{array}{lll}
\varepsilon_{\mathrm{p}} \neq 0, \quad \varepsilon_{\mathrm{S}}=0 & \text { for } t \leqq 0, \\
& (\text { period } \mathrm{A}) \\
\varepsilon_{\mathrm{p}} \neq 0, \quad \varepsilon_{\mathrm{s}} \neq 0 & \text { for } 0 \leqq t \leqq t_{1}, \\
& & (\text { period } \mathrm{B}) \\
\varepsilon_{\mathrm{p}} \neq 0, \quad \varepsilon_{\mathrm{S}}=0 & \text { for } t \geqq t_{1} . \\
& & (\text { period } \mathrm{C})
\end{array}
$$

To obtain solutions of (2) for the different periods of time, both signal and pump microwave radiation have been assumed to be resonant with the pump and signal transition respectively, giving with $(4 a-c)$

$$
\Delta \omega_{\mathrm{p}}=0, \Delta \omega_{\mathrm{s}}=0, \Delta \omega=0 . \quad(6 \mathrm{a}-\mathrm{c})
$$

For further simplification some approximations have been made with respect to the relaxation parameters in (2). Firstly, identical coherence decay times $\left(T_{2}^{\mathrm{ab}}=T_{2}^{\mathrm{bc}}=T_{2}^{\mathrm{ac}}\right)$ have been assumed as was found experimentally for the considered system [13], [14], and [15]. Secondly, cross-relaxation between the variables $W_{\mathrm{s}}$ and $W_{\mathrm{p}}$ representing population differences may be neglected with respect to $T_{1}$ relaxation of $W_{\mathrm{p}}$ and $W_{\mathrm{s}}$ itself [10] $\left(\gamma_{\mathrm{w}}, \gamma_{z} \ll \gamma_{x}, \gamma_{y}\right)$ which is assumed to be described by identical $T_{1}$ relaxation times [16] $\left(\gamma_{x}=\gamma_{y}\right)$. Finally, by using the experimental findings of a rather small difference between $T_{1}$ - and $T_{2}$-relaxation times for OCS two level systems [13-15], [16], we may approximate

$$
\alpha=1 / T_{2}^{i j}=\gamma_{x}=\gamma_{y} \quad(i j=\mathrm{ab}, \mathrm{bc}, \mathrm{ac}) .
$$

Although this approximation does not hold rigorously, the result obtained with it presents the most important features of the solutions under consideration.

With the approximations given above, the solutions of (2) are found straightforwardly for the different periods of time. To obtain the initial conditions at the time $t=0$, we may use the steadystate solutions of (2) for period A (no signal microwave for times $t<0$ which are long with respect to the relaxation times), giving with (5a), (6) and (7)

$$
\begin{aligned}
& U_{\mathrm{s}}(0)=V_{\mathrm{s}}(0)=U_{\mathrm{p}}(0)=U(0)=V(0)=0, \\
& V_{\mathrm{p}}(0)=-\alpha x_{\mathrm{p}} W_{\mathrm{p}}^{0} /\left(\alpha^{2}+x_{\mathrm{p}}^{2}\right) \\
& W_{\mathrm{p}}(0)=\alpha^{2} W_{\mathrm{p}}^{0} /\left(\alpha^{2}+x_{\mathrm{p}}^{2}\right) \\
& W_{\mathrm{s}}(0)=W_{\mathrm{s}}^{0}+x_{\mathrm{p}}^{2} W_{\mathrm{p}}^{0} /\left(\alpha^{2}+x_{\mathrm{p}}^{2}\right) . \quad(8 \mathrm{a}-\mathrm{d})
\end{aligned}
$$


For sufficiently high pump power, $x_{\mathrm{p}} \gg \alpha$, we have

$$
\begin{aligned}
& V_{\mathrm{p}}(0) \approx 0, \quad W_{\mathrm{p}}(0) \approx 0, \\
& W_{\mathrm{s}}(0) \approx W_{\mathrm{s}}^{0}+W_{\mathrm{p}}^{0} / 2,
\end{aligned}
$$

i.e. saturation of the pump transition.

For period $\mathrm{B}\left(0 \leqq t \leqq t_{1}\right)$ we may neglect the collisional damping terms in (2) for a signal pulse duration which is short with respect to the relaxation times $\left(t_{1} \ll 1 / \alpha\right)$. We then obtain with the initial conditions (8), (9) at $t=0$ the following solutions for $0 \leqq t \leqq t_{1}$

$$
\begin{aligned}
U_{\mathrm{s}}(t)=0 & , \\
V_{\mathrm{s}}(t)=- & \left(x_{\mathrm{s}} / 8 \Omega^{3}\right) W_{\mathrm{s}}(0) \\
& \cdot\left\{2 x_{\mathrm{p}}^{2} \sin \Omega t+x_{\mathrm{s}}^{2} \sin 2 \Omega t\right\}, \\
W_{\mathrm{s}}(t)= & \left(1 / 8 \Omega^{4}\right) W_{\mathrm{s}}(0) \\
& \cdot\left\{x_{\mathrm{p}}^{2} x_{\mathrm{s}}^{2} \cos \Omega t+\left(x_{\mathrm{p}}^{2} x_{\mathrm{s}}^{2} / 4+x_{\mathrm{s}}^{4} / 2\right)\right. \\
& \left.\cdot \cos 2 \Omega t+x_{\mathrm{p}}^{4} / 2-x_{\mathrm{p}}^{2} x_{\mathrm{s}}^{2} / 4\right\}, \\
U_{\mathrm{p}}(t)=0, & \quad(10) \\
V_{\mathrm{p}}(t)=-\left(x_{\mathrm{p}} x_{\mathrm{s}}^{2} / 8 \Omega^{3}\right) W_{\mathrm{s}}(0) & \cdot\{2 \sin \Omega t-\sin 2 \Omega t\}, \\
W_{\mathrm{p}}(t)= & \left(x_{\mathrm{s}}^{2} / 8 \Omega^{4}\right) W_{\mathrm{s}}(0) \\
& \cdot\left\{x_{\mathrm{p}}^{2} \cos \Omega t-\left(x_{\mathrm{p}}^{2} / 2+x_{\mathrm{s}}^{2} / 4\right) \cos 2 \Omega t\right. \\
& \left.\quad-x_{\mathrm{p}}^{2} / 2+x_{\mathrm{s}}^{2} / 4\right\}, \\
U(t)= & \left(x_{\mathrm{p}} x_{\mathrm{s}} / 8 \Omega^{4}\right) W_{\mathrm{s}}(0) \\
& \cdot\left\{\left(x_{\mathrm{p}}^{2}-x_{\mathrm{s}}^{2}\right) \cos \Omega t+\left(x_{\mathrm{s}}^{2} / 2\right) \cos 2 \Omega t\right. \\
& \left.\quad+x_{\mathrm{s}}^{2} / 2-x_{\mathrm{p}}^{2}\right\}, \\
V(t)=0 & , \quad \text { where } \quad \Omega^{2}=\left(x_{\mathrm{p}}^{2}+x_{\mathrm{s}}^{2}\right) / 4 .
\end{aligned}
$$

It may be noted that the transient double resonance solutions (10) for the time dependencies of the polarization components in quadrature to the external MW fields $\left(\sim V_{\mathrm{S}}\right.$ and $\left.\sim V_{\mathrm{p}}\right)$ and of the population differences $\left(\sim W_{\mathrm{s}}\right.$ and $\left.\sim W_{\mathrm{p}}\right)$ are more complicated than for the single resonance two-level case. In particular, no simple condition for optimum polarization of the sample gas (such as a $\pi / 2$-pulse) may be formulated for the double resonance case. In addition to polarization and population difference, a nonzero coherence between the levels $E_{\text {a }}$ and $E_{\mathrm{c}}$ $(\sim U)$ is created which may be ascribed to a transient two-photon double resonance effect.

At the time $t=t_{1}$ the signal microwave radiation is switched off and transient solutions of (2) for $t \geqq t_{1}$ (period C) may be obtained for $\varepsilon_{\mathrm{S}}=0$ and the initial conditions $(10)$ at $t=t_{1}$. We are interested here only in the solutions for $U_{\mathrm{s}}$ and $V_{\mathrm{s}}$ which are related to the observable quantities in our experi- ments (see below), given by

$$
\begin{aligned}
U_{\mathrm{s}}\left(t^{\prime}\right) & =0, \\
V_{\mathrm{s}}\left(t^{\prime}\right) & =\exp \left(-\alpha t^{\prime}\right)\left\{V_{\mathrm{s}}\left(t_{1}\right) \cos \left(x_{\mathrm{p}} / 2\right) t^{\prime}\right. \\
& \left.-U\left(t_{1}\right) \sin \left(x_{\mathrm{p}} / 2\right) t^{\prime}\right\},
\end{aligned}
$$

where $t^{\prime}=t-t_{1}$

To obtain the polarization $P$ of the sample gas which may be observed in the transient and steadystate regime, we used the equation of definition for $P$

$$
P=N \operatorname{Tr}(\mu \varrho),
$$

where $N$ is the number density of molecules, $\varrho$ the density matrix and $\mu$ the matrix of the dipole moment operator. Using the real-valuedness of the transition dipole matrix elements, we then obtain, using the transformation of $\varrho$ into the interaction representation [17], and $(3 \mathrm{a}-\mathrm{d})$

$$
\begin{aligned}
P(t)= & N \mu_{\mathrm{ab}} U_{\mathrm{s}}(t) \cos \omega_{\mathrm{s}} t-N \mu_{\mathrm{ab}} V_{\mathrm{s}}(t) \sin \omega_{\mathrm{s}} t \\
& +N \mu_{\mathrm{bc}} U_{\mathrm{p}}(t) \cos \omega_{\mathrm{p}} t \\
& -N \mu_{\mathrm{bc}} V_{\mathrm{p}}(t) \sin \omega_{\mathrm{p}} t .
\end{aligned}
$$

In our experimental arrangement, microwave radiation with frequency components close to $\omega_{\mathrm{p}}$ is blocked from reaching the detection system (see Fig. 2) and therefore not observed. Consequently only the first two terms in (13) have to be considered further (slowly varying amplitude functions assumed). The detected microwave field which is emitted by the molecular sample gas at times $t \geqq t_{1}$ is then proportional to the polarization [18]

$$
\begin{aligned}
P\left(t^{\prime}\right)=N \mu_{\mathrm{ab}} & \left\{U_{\mathrm{s}}\left(t^{\prime}\right) \cos \omega_{\mathrm{s}} t^{\prime}\right. \\
& \left.-V_{\mathrm{s}}\left(t^{\prime}\right) \sin \omega_{\mathrm{s}} t^{\prime}\right\}
\end{aligned}
$$

which may be rewritten with (11)

$$
\begin{aligned}
P\left(t^{\prime}\right)=- & \left(N \mu_{\mathrm{ab}} / 2\right) \exp \left(-\alpha t^{\prime}\right) \\
& \cdot\left\{V _ { \mathrm { s } } ( t _ { 1 } ) \left(\sin \left(\omega_{\mathrm{s}}+x_{\mathrm{p}} / 2\right) t^{\prime}\right.\right. \\
& \left.+\sin \left(\omega_{\mathrm{s}}-x_{\mathrm{p}} / 2\right) t^{\prime}\right) \\
& +U\left(t_{1}\right)\left(\cos \left(\omega_{\mathrm{s}}+x_{\mathrm{p}} / 2\right) t^{\prime}\right. \\
& \left.\left.-\cos \left(\omega_{\mathrm{s}}-x_{\mathrm{p}} / 2\right) t^{\prime}\right)\right\} .
\end{aligned}
$$

Equation (15) represents the superposition of two decaying signals oscillating at the frequencies $\omega_{\mathrm{s}}+x_{\mathrm{p}} / 2$ and $\omega_{\mathrm{s}}-x_{\mathrm{p}} / 2$ respectively. With Fourier transformation from the time to the frequency domain we then have a superposition of two Lorentzian lines with center peaks at $\omega_{1 / 2}=\omega_{\mathrm{s}} \pm x_{\mathrm{p}} / 2$ as observed in our experiments. According to (4e) the line splitting is pump power dependent and given by

$$
\omega_{1}-\omega_{2}=\left(2 \mu_{\mathrm{bc}} / \hbar\right) \varepsilon_{\mathrm{p}} .
$$


This result is in agreement with the familiar coherence splitting of double resonance lines which have been predicted earlier [11] in a description of steadystate double resonance experiments under the condition of high pump power and low probe microwave power.

\section{Conclusion}

In view of the many possible modifications and applications we hope that the Fourier transform

[1] A. Battaglia, A. Gozzini, and E. Polacco, Nuovo Cim. 14, 1076 (1959).

[2] T. Yajima and K. Shimoda, J. Phys. Soc. Japan 15, $1968(1960)$.

[3] T. Yajima, J. Phys. Soc. Japan 16, 1709 (1961).

[4] A. P. Cox, G. W. Flynn, and E. B. Wilson, J. Chem. Phys. 42, 3094 (1965).

[5] G. Bestmann, H. Dreizler, H. Mäder, and U. Andresen, Z. Naturforsch. 35a, 392 (1980).

[6] G. Bestmann and H. Dreizler, Z. Naturforsch. 37a, 58 (1982).

[7] G. Bestmann and H. Dreizler, Z. Naturforsch. 37a, 615 (1982).

[8] G. Bestmann, H. Dreizler, E. Fliege, and W. Stahl, to be published.

[9] G. Maki, J. Phys. Ref. Data 3, 221 (1974). double resonance can be developed to an as useful tool as double resonance already is in normal microwave spectroscopy.

\section{Acknowledgement}

We thank the workshop of our institute, the members of our group for help and discussions and the Deutsche Forschungsgemeinschaft and Fonds der Chemie for funds.

[10] C. Feuillade, J. G. Baker, and C. Bottcher, Chem. Phys. Lett. 40, 121 (1976).

[11] A. Javan, Phys. Rev. 107, 1579 (1957).

[12] W. K. Liu and R. A. Marcus, J. Chem. Phys. 63, 272 (1975).

[13] S. L. Coy, J. Chem. Phys. 63, 5145 (1975).

[14] W. E. Hoke, D. R. Bauer, J. Ekkers, and W. H. Flygare, J. Chem. Phys. 64, 5276 (1976).

[15] H. Bomsdorf and H. Dreizler, Z. Naturforsch. 36a, 473 (1981).

[16] H. Mäder, Z. Naturforsch. 34a, 1170 (1979).

[17] W. Schrepp and H. Mäder, Chem. Phys. 63, 113 (1981).

[18] T. G. Schmalz and W. H. Flygare, Laser and Coherence Spectroscopy, p. 125ff., Plenum Press, New York 1978. 